\title{
Tight-binding study of nonmagnetic-defect-induced magnetism in graphene
}

\author{
H. Kumazaki and D.S. Hirashima \\ Department of Physics, Nagoya University, Nagoya 464-8602, Japan \\ E-mail: dai@slab.phys.nagoya-u.ac.jp
}

Received March 3, 2008

\begin{abstract}
This review describes a study of nonmagnetic-defect-induced magnetism of graphene on the basis of a tight-binding model. A vacancy induces a quasilocalized impurity state at the chemical potential around itself, and it leads to formation of local magnetic moments. Connection between a quasilocalized state around a vacancy and the edge localized states near a zigzag edge is studied in detail. Magnetism associated with many vacancies and edge structures is also reviewed. Some new results of magnetism associated with many vacancies are presented.
\end{abstract}

PACS: 75.75.+a Magnetic properties of nanostructures;

73.20.At Surface states, band structure, electron density of states;

75.70.Ak Magnetic properties of monolayers and thin films.

Keywords: graphene, magnetism, defects, edges.

\section{Introduction}

The recent discovery of monolayer graphite [1] monolayer graphene - has triggered a surge of intensive research of this unique material. In addition to the unique physics of massless Dirac fermions realized in graphene, the potentiality in future nanoelectronics applications is the driving force of the research effort [2]. Applications in electronic devices taking advantage of spin degrees of freedom - spintronics applications - are also discussed [3].

Magnetism in carbon allotropes has indeed been a fundamental and also controversial problem for a long time [4-7]. Apart from magnetic impurities, the defect-induced mechanism is the most probable mechanism of magnetism in carbon-based materials. Indeed, defect-induced magnetism has been theoretically studied in various carbon allotropes extensively [8-34]. The simplest mechanism is the one caused by free dangling bonds associated with vacancies. A dangling bond has a net magnetic moment, which may induce a magnetic state. Even if a defect itself is nonmagnetic, it can induce local magnetism of $\pi$ electrons around itself. A well-known example is the localized edge magnetism associated with a (hydrogenterminated) zigzag edge of graphene [8] or a carbon nanotube [12].
Graphene is the building block of carbon allotropes, graphite, carbon nanotubes, and fullerenes. Therefore, it is important to study the possible magnetism of graphene to understand magnetism in other carbon allotropes. Nonmagnetic-defect-induced magnetism in graphene has also been reported using first-principles calculations [16-24]. We have also studied the problem using a tight-binding model, and also found nonmagnetic-defect-induced magnetism in graphene [22,26-28,31]. Here, we review these studies, and show that the origin of the local magnetism is the impurity state induced by a vacancy introduced on a honeycomb lattice [22,35-37] and that the sublattice configuration of vacancies matters when more than one vacancies are introduced. The latter fact is a manifestation of Lieb's theorem on magnetism on a bipartite lattice [38]. We then argue that the edge magnetism on a zigzag edge of graphene [8] can be naturally understood as accumulation of vacancy-induced local magnetism.

In the next section, we introduce a tight-binding model of graphene and discuss an impurity state induced by a vacancy (nonmagnetic defect). Adding the Coulomb interaction to the Hamiltonian, we review the possible local magnetism induced by a vacancy (vacancies) in Sec. 3. In Sec. 3, we also present some new results of local moment formation induced by extended vacancies. We then briefly review the magnetism on edges with various structures 
in Sec. 4, and the last section is devoted to a summary of the paper.

\section{Quasilocalized states induced by vacancies}

We consider $\pi$ electrons of graphene, which can be described by a tight-binging model on a honeycomb lattice (Fig. 1)

$$
\begin{aligned}
\mathcal{H}_{0} & =-t \sum_{\left(\mathbf{n A}, \mathbf{n}^{\prime} B\right), \sigma}\left[c_{\mathbf{n} A \sigma}^{\dagger} c_{\mathbf{n}^{\prime} B \sigma}+\text { h.c. }\right]= \\
& =\sum_{\mathbf{k}, \sigma}\left[\varepsilon(\mathbf{k}) c_{\mathbf{k A \sigma} \sigma}^{\dagger} c_{\mathbf{k} B \sigma}+\text { h.c. }\right],
\end{aligned},
$$

where we consider only the electron transfer $t$ between the nearest neighbor site pairs $\left(\mathbf{n A}, \mathbf{n}^{\prime} B\right)$; $\mathbf{n}$ and $\mathbf{n}^{\prime}$ represent the positions of unit cells, and $A$ and $B$ stand for the sublattice indices. $c_{\mathbf{n} \alpha \sigma}\left(c_{\mathbf{n} \alpha \sigma}^{\dagger}\right)$ is an annihilation (creation) operator of a $\pi$ electron with spin $\sigma$ at the site $(\mathbf{n} \alpha)$, and $c_{\mathbf{k} \alpha \sigma}\left(c_{\mathbf{k} \alpha \sigma}^{\dagger}\right)$ is its Fourier transformation. Using the vectors $\tau_{j}(j=1,2,3)$ connecting a site on sublattice $A$ and the three nearest neighbor sites on sublattice $B$, we can write

$$
\varepsilon(\mathbf{k})=-t \sum_{j=1}^{3} \mathrm{e}^{i \mathbf{k} \cdot \tau_{j}} .
$$

The band dispersion is given by $E(\mathbf{k})=\left|E_{ \pm}(\mathbf{k})= \pm\right| \varepsilon(\mathbf{k})$. It vanishes at points $\mathrm{K}(2 \pi / 3 a, 2 \pi / \sqrt{3} a)$ and $\mathrm{K}^{\prime}(4 \pi / 3 a, 0)$, and has a linear dispersion around those points (a massless Dirac fermion),

$$
E_{ \pm}(\mathbf{k})= \pm \gamma \delta k
$$

where $\delta \mathbf{k}$ is the deviation of $\mathbf{k}$ from $\mathrm{K}$ (or $\mathrm{K}^{\prime}$ ), $\gamma=\sqrt{3} t a / 2$, and $a$ is the lattice constant, $a=2.46 \AA$.

We denote the number of unit cells by $N_{u} ; N_{u}=L^{2}$ where $L$ is the linear size of the lattice. Without vacancies, the number of the lattice points $N_{S}$ is $N_{s}=2 N_{u}$. We restrict ourselves to the half-filled case where the electron

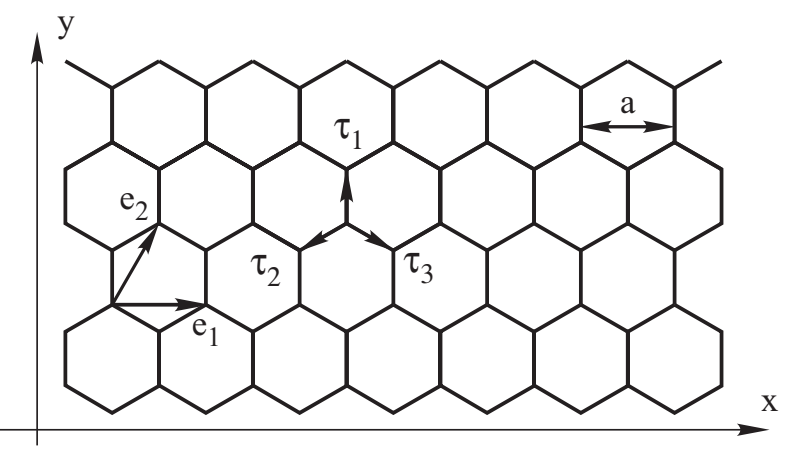

Fig. 1. Honeycomb lattice. The primitive vectors are $\mathbf{e}_{1}$ and $\mathbf{e}_{2}$, whose magnitude is $a,\left|\mathbf{e}_{1}\right|=\left|\mathbf{e}_{2}\right|=a$. Three vectors connecting the nearest neighbor sites are denoted by $\tau_{j}(j=1,2$, and 3$)$, $\left|\tau_{j}\right|=a / \sqrt{3}$. number $N_{\mathrm{e}}$ is equal to the site number $N_{s}, N_{e}=N_{s}$, even in the presence of vacancies.

In this paper, we implicitly assume that all dangling bonds are terminated appropriately, e.g., by hydrogen atoms. We consider magnetism caused by $\pi$ electrons.

Now we introduce impurity potentials. A short-range impurity potential at $(\mathbf{n} \alpha)$ is expressed by

$$
\mathcal{H}(\mathbf{n} \alpha)=u \sum_{\sigma} c_{\mathbf{n} \alpha \sigma}^{\dagger} c_{\mathbf{n} \alpha \sigma} .
$$

The limit of $u \rightarrow \infty$ simulates a strong impurity such as a vacancy, although $u=\infty$ may be unrealistic for actual vacancies. The opposite limit $u \rightarrow-\infty$ may simulate the effect of a hydrogen chemisorption site. In both limits, $N_{s}=2 N_{u}-N_{i}$, where $N_{i}$ is the number of impurities.

First, we briefly study the case with a single impurity [35]. For the moment, we suppress the spin suffixes. Expanding a single electron wave function $\Phi(\mathbf{r})$ with the Wannier functions $\phi_{\mathbf{n} \alpha}(\mathbf{r}), \Phi(\mathbf{r})=\sum_{\mathbf{n}, \alpha} C_{\mathbf{n} \alpha} \phi_{\mathbf{n} \alpha}(\mathbf{r})$, we can write down the Schrödinger equation in the presence of an impurity at $\mathbf{r}=\mathbf{r}_{0}$ on sublattice $\mathrm{A}$,

$$
\begin{aligned}
& \left(\begin{array}{cc}
E & -\varepsilon(\mathbf{k}) \\
-\varepsilon(\mathbf{k})^{*} & E
\end{array}\right)\left(\begin{array}{l}
C_{\mathbf{k} A} \\
C_{\mathbf{k} B}
\end{array}\right)= \\
& =\frac{u \mathrm{e}^{-i \mathbf{k} \cdot \mathbf{r}_{0}}}{N_{u}} \sum_{\mathbf{k}^{\prime}}\left(\begin{array}{cc}
\mathrm{e}^{i \mathbf{k}^{\prime} \cdot \mathbf{r}_{0}} & 0 \\
0 & 0
\end{array}\right)\left(\begin{array}{l}
C_{\mathbf{k}^{\prime} A} \\
C_{\mathbf{k}^{\prime} B}
\end{array}\right),
\end{aligned}
$$

from which we obtain the eigenvalue equation for a bound state,

$$
\frac{u}{N_{u}} \sum_{\mathbf{k}} \frac{E}{E^{2}-|\varepsilon(\mathbf{k})|^{2}}=1 .
$$

In the limit of $u \rightarrow \infty$, we have $E \simeq \varepsilon_{c}^{2} /\left[u \ln \left(\varepsilon_{c} / u\right)\right] \rightarrow 0$, where $\varepsilon_{c}$ is a cutoff energy, that is, the eigenenergy vanishes. This means that an impurity state is induced at the chemical potential $\mu=0$ by a strong impurity potential. Putting $E=0$, we readily have $C_{\mathbf{k} A}=0$, which also means that $C_{\mathbf{n} A}=0$. The amplitude of this wave function vanishes on sublattice $\mathrm{A}$, the same sublattice where a vacancy is. On the other hand, we have

$$
C_{\mathbf{k} B}=\mathcal{N} \frac{\mathrm{e}^{-i \mathbf{k} \cdot \mathbf{r}_{0}}}{\sqrt{N_{u}} \varepsilon(\mathbf{k})},
$$

where $\mathcal{N}=-\lim _{u \rightarrow \infty} u C_{0 A}$ is a normalization constant. We then obtain $u \rightarrow \infty$

$$
C_{\mathbf{n} B}=\frac{1}{\sqrt{N_{u}}} \sum_{\mathbf{k}} \mathrm{e}^{i \mathbf{k} \cdot \mathbf{r}_{n}} C_{\mathbf{k} B}=\mathcal{N} D\left(\mathbf{r}_{n}-\mathbf{r}_{0}, \tau_{1}\right),
$$

with

$$
D(\mathbf{r}, \tau)=\frac{1}{N_{u}} \sum_{\mathbf{k}} \frac{\mathrm{e}^{i \mathbf{k} \cdot(\mathbf{r}+\tau)}}{\varepsilon(\mathbf{k})},
$$


where $\tau$ is the vector connecting the two lattice points in a unit cell.

We can show that $D(\mathbf{r}, \tau) \propto 1 / r$ at $r>>a$. This means that the wave function is localized around the impurity site, but not strongly enough to make the normalization constant $\mathcal{N}$ independent of the system size. This is why we call this state a quasilocalized state. The normalization constant $\mathcal{N} \propto 1 / \sqrt{\ln L}$, if we normalize the wave function so that $\int \mathrm{d} \mathbf{r} \Phi(\mathbf{r})^{2}=1$.

In the limit of $u \rightarrow \infty$, the number of states in the valence band $(E<0)$ is $N_{u}-1$ and that in the conduction band $(E>0)$ is also $N_{u}-1$. The strong impurity potential induces a quasilocalized state just at $E=0$ as shown above, in addition to pushing a state to positive infinity. Then, in the ground state at the half-filling $N_{e}=N_{L}=$ $=2 N_{u}-1$, either up-spin or a down-spin occupies the quasilocalized state at $E=0$, generating (quasi)localized magnetic moments around a vacancy even in the absence of the Coulomb interaction. It is simply natural that the ground state has a net magnetic moment, because the number of electrons $N_{e}$ is odd. The point is that the magnetic moments are not spread over the system, but are (quasi)localized around a vacancy, which is specific for the honeycomb lattice.

Next, we consider cases with two impurities [26]. First, the case with two impurities at $\mathbf{r}=\mathbf{r}_{0}$ and $\mathbf{r}_{1}$ on the same sublattice $A$ is considered. An eigenvalue equation for a bound state is also easily found, and is given by

$$
\frac{u}{N_{u}} \sum_{\mathbf{k}} \frac{E}{E^{2}-|\varepsilon(\mathbf{k})|^{2}}\left(1+\frac{C_{1 A}}{C_{0 A}} \mathrm{e}^{i \mathbf{k} \cdot \cdot\left(\mathbf{r}_{0}-\mathbf{r}_{1}\right)}\right)=1
$$

from which we readily see that the eigenvalue $E$ vanishes as $|u| \rightarrow \infty$. The wave function also vanishes on sublattice $A, C_{\mathbf{k} A}=C_{\mathbf{n} A}=0$, and

$$
C_{\mathbf{n} B}=\mathcal{N}_{0} D\left(\mathbf{r}_{n}-\mathbf{r}_{0}, \tau_{1}\right)+\mathcal{N}_{1} D\left(\mathbf{r}_{n}-\mathbf{r}_{1}, \tau_{1}\right),
$$

where $\mathcal{N}_{i}$ is a normalization constant. This means that a quasilocalized state associated with one vacancy is not affected by the other vacancy, the wave function is simply an arbitrary linear combination of two quasilocalized states associated with two vacancies, and the two states remain degenerate at $E=0$.

At the half-filling $N_{e}=N_{L}=2 N_{u}-2$, two electrons occupy those two degenerate states. Without the Coulomb interaction, a singlet state and triplet states are degenerate, and Lieb's theorem guarantees that the triplet states become the ground state in the presence of the Hubbard $U(>0)$ [38]. As we consider only the nearest neighbor electron transfer integral, the lattice is bipartite. The realization of triplet states is also consistent with Hund's rule.

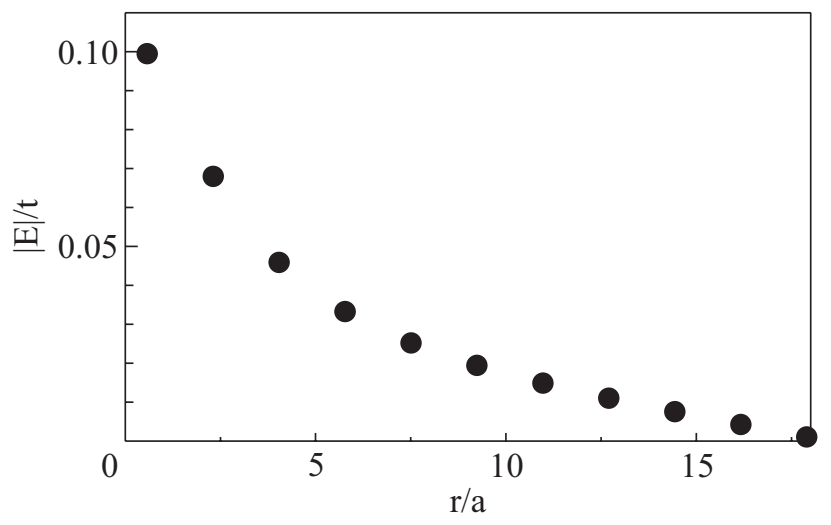

Fig. 2. Energy $E$ of a vacancy state induced when two vacancies are introduced on the different sublattices as a function of the distance $r$ between the two vacancies.

When two impurities are on the different sublattices, the situation is different. We can see that the eigenvalue $E$ does not vanish in general. It vanishes only when the distance $r$ between two vacancies goes to infinity (Fig. 2); $E \propto 1 / r$ [26]. This is simply because there occurs a «conflict» between the two vacancies: One vacancy on sublattice $A$ requires the wave function to be finite only on sublattice $B$, and the other vacancy on sublattice $B$ requires the opposite. This leads to the effective repulsion between the two quasilocalized states associated with vacancies on different sublattices, and they are no more at $E=0$.

The reduction in the density of states due to the repulsion between the quasilocalized states is unfavorable for local moment formation. Lieb's theorem states that the ground state must be singlet (at a finite $U$ ) in the present case, where the number of lattice points on sublattice $A$ is equal to that on sublattice $B$ [38]. We denote the number of vacancies on sublattice $A$ by $N_{A}$, and that on sublattice $B$ by $N_{B}$. When $N_{A}=N_{B}$, the number of lattice points on sublattice $A$ is equal to that on sublattice $B$.

When $N_{A}=N_{B}$ and the ground state is a singlet one, there are still two possibilities [28,29]: One is that no moment is formed at any sites. The other possibility is that a magnetic moment is locally generated around each vacancy and the two local moments are coupled to form a spin singlet state. Which is realized depends on the configuration of two vacancies, the strength in interaction, etc, which will be studied in the next section.

The results obtained for two vacancies can be straightforwardly extended to the case with more vacancies. For example, $N$ vacancies on the same sublattice generate $N$-fold degenerate (quasilocalized) states at $E=0$. It is interesting to study the case where vacancies are in a row on the same sublattice, say sublattice $A$ (Fig. 3 ). These vacancies generate a zigzag edge and a Klein edge. Assuming the periodic boundary condition along the $x$-direc- 


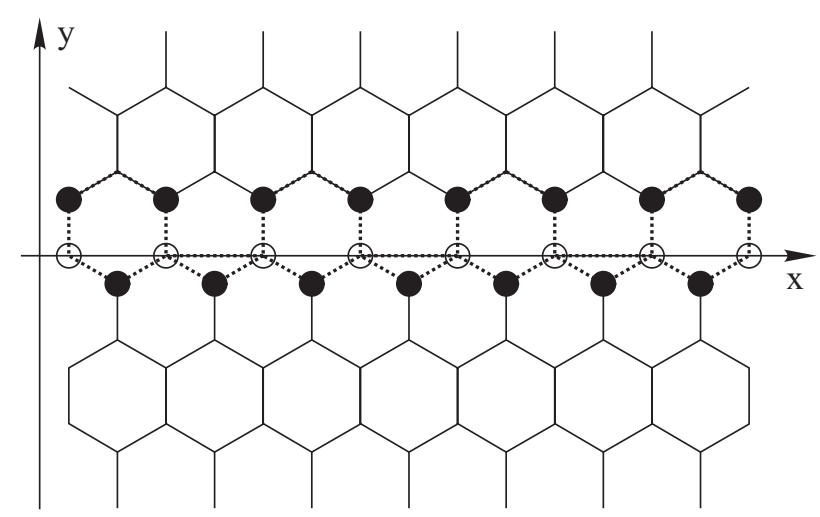

Fig. 3. Removing carbon atoms (open dots) in a row from the same sublattice generates a zigzag edge and a Klein edge. Edge sites on both edges are represented by closed dots.

tion, the direction along the edges, the wavevector $p$ (along the $x$-direction) is a good quantum number. The wave function $C_{\mathbf{n} B}(p)$ is then expressed as

$$
C_{\mathbf{n} B}(p) \propto \sum_{\ell} \mathrm{e}^{i p \ell a} D\left(\mathbf{r}_{n}-\mathbf{r}_{\ell}, \tau_{1}\right) \propto \mathrm{e}^{i p x_{n}} d_{p}\left(y_{n}\right),
$$

where

$$
\mathbf{r}_{n}=\left(x_{n}, y_{n}\right), \mathbf{r}_{\ell}=\left(x_{\ell}, 0\right)=(\ell a, 0),
$$

and

$$
d_{p}\left(y_{n}\right)=\sum_{k_{y}} \frac{\mathrm{e}^{i k_{y} a / \sqrt{3}}}{\varepsilon\left(p, k_{y}\right)} \mathrm{e}^{i k_{y} y_{n}} ;
$$

the region of the summation of $k_{y}$ is shown in Fig. 4. As the summand $\mathrm{e}^{i k_{y} a / \sqrt{3}} / \varepsilon\left(p, k_{y}\right)$ is a periodic function of $k_{y}$ (of period $\left.4 \pi /(\sqrt{3} a)\right), d_{p}\left(y_{n}\right)$ decreases faster than any power of $1 / y_{n}$ as $y_{n} \rightarrow \infty$, except for at $p=2 \pi /(3 a)$ (or $4 \pi /(3 a))$, where $\varepsilon\left(p, k_{y}\right)$ vanishes at $k_{y}=2 \pi /(\sqrt{3} a)$ (or 0 ). This is indeed what is found by Fujita et al. (see also below) [8].

Although each vacancy generates a quasilocalized state whose amplitude decreases proportionally to the inverse of the distance from the position of the vacancy, those vacancies in a row on the same sublattice generate exponentially localized states (plus extended states at $p= \pm 2 \pi /(3 a))$ as a results of interference. Note also that those extended states may be important for coupling between magnetic moments on both edges of a graphene ribbon with zigzag edges [25].

Strictly speaking, what we have shown is not exactly the same as the localized states found by Fujita et al. They studied the case with a graphene ribbon with two zigzag edges. In this case, the edge localized states exist only at $2 \pi / 3<p a<4 \pi / 3$ (i.e., the number of the localized states is one third of the number of vacancies). The result obtained above corresponds to a graphene ribbon with a zig-

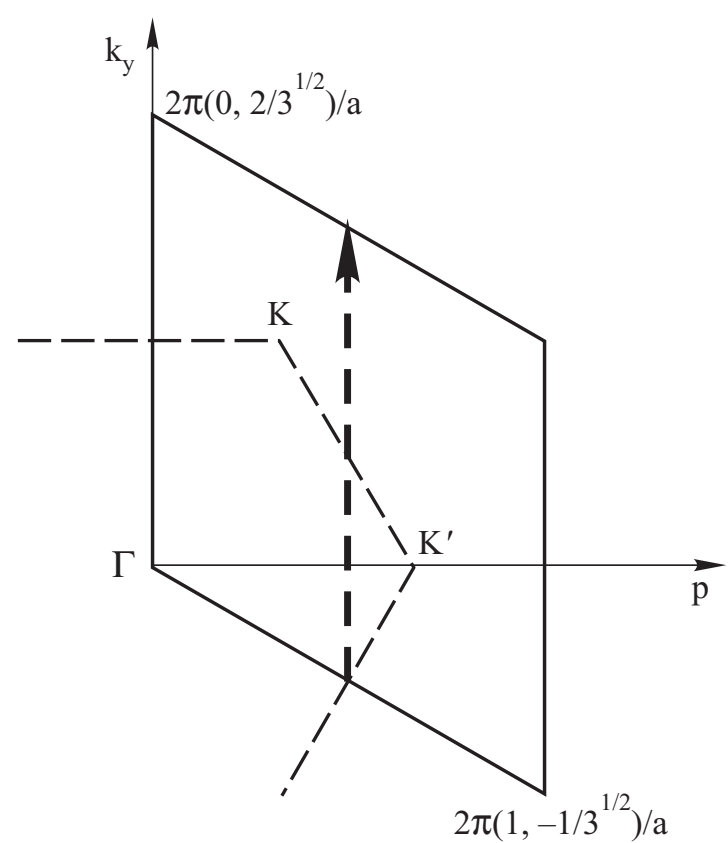

Fig. 4. The first Brillouin zone of a honeycomb lattice (the rhombus), and the thick dashed line shows the integral region of $k_{y}$ in Eq. (14). When $p=2 \pi /(3 a)$ or $4 \pi /(3 a)$, the line passes over $\mathrm{K}\left(2 \pi /(3 a), 2 \pi /(\sqrt{3} a)\right.$ or $\mathrm{K}^{\prime}(4 \pi /(3 a), 0)$, where $\varepsilon\left(p, k_{y}\right)$ vanishes.

zag edge and a Klein edge, where it was shown that the edge localized states exist for any $p$ (i.e., the number of the localized states is the same as that of vacancies) [39].

\section{Vacancy-induced magnetism}

Now we proceed to study magnetism induced by nonmagnetic defects. To do so, we have to introduce the Coulomb interaction between $\pi$ electrons. We consider the local Coulomb interaction $U$,

$$
\mathcal{V}=U \sum_{\mathbf{n} \alpha, \sigma} n_{\mathbf{n} \alpha \uparrow} n_{\mathbf{n} \alpha \downarrow} .
$$

We thus consider a Hubbard model on the honeycomb lattice to study magnetism of graphene. We resort to a mean field approximation to treat $\mathcal{V}$,

$$
\mathcal{V} \rightarrow U \sum_{\mathbf{n}, \alpha, \sigma}\left\langle n_{\mathbf{n} \alpha-\sigma}\right\rangle n_{\mathbf{n} \alpha \sigma} .
$$

By diagonalizing the mean-field approximated hamiltonian on a finite size lattice $(L \times L)$, we can self-consistently determine the charge density $n_{\mathbf{n} \alpha}=\left\langle n_{\mathbf{n} \alpha} \uparrow+n_{\mathbf{n} \alpha \downarrow}\right\rangle$ and the spin density $m_{\mathbf{n} \alpha}=\left\langle n_{\mathbf{n} \alpha} \uparrow-n_{\mathbf{n} \alpha \downarrow}\right\rangle$ at each lattice point. In actual calculations, we use $U=1.2 t$ unless otherwise stated. In the mean field approximation, the ground state of the half-filled honeycomb lattice is an antiferromagnetic state at $U>U_{\text {cr }}=2.23 t$.

It was shown in a previous study that the ferrimagnetic order is induced around the vacancy [27]. Without $U$, only 
the density of one spin component (up or down) is finite because the wave function of the quasilocalized state is finite only on one sublattice. The interaction $U$ makes the minor spin component develop, i.e., it enhances the staggered spin moment. At $U=1.2 t$, the total staggered moment

$$
M_{s ; \text { tot }}=\left|\sum_{\mathbf{n}}\left(m_{\mathbf{n} A}-m_{\mathbf{n} B}\right)\right|
$$

is $M_{s ; \text { tot }} \simeq 2.2$ (for $L=32$ ). The total moment

$$
M_{\text {tot }}=\left|\sum_{\mathbf{n}}\left(m_{\mathbf{n} A}+m_{\mathbf{n} B}\right)\right|
$$

is evidently $M_{\text {tot }}=1\left(S_{z}=1 / 2\right)$. The nonmagnetic-defect-induced magnetic moments were also obtained by first-principles calculations [16,24].

As mentioned in the previous chapter, the ground state is a singlet one when two vacancies are introduced on the different sublattices, $A$ and $B$. However, it is still possible that local magnetic moments are induced around each vacancy $[28,29]$. Figure 5 shows the dependence of the total sublattice magnetization $M_{s \text {; tot }}$ on the distance $r$ between two vacancies put on the different sublattices. When the vacancies are close to each other, no local magnetic moments are induced. When they separate apart, local moments develop around each vacancy. The magnitude of the total staggered moment approaches to 4.4 , twice the staggered moment in the case with a single vacancy. The moments couple antiferromagnetically, and the ground state is a singlet one, but the coupling weakens as they separate apart.

When the two vacancies are introduced on the same sublattice, the quasilocalized states remain at the chemical potential, and local moments are induced around both vacancies. They couple ferromagnetically, and the ground state is a triplet one in harmony with Lieb's theorem [38].

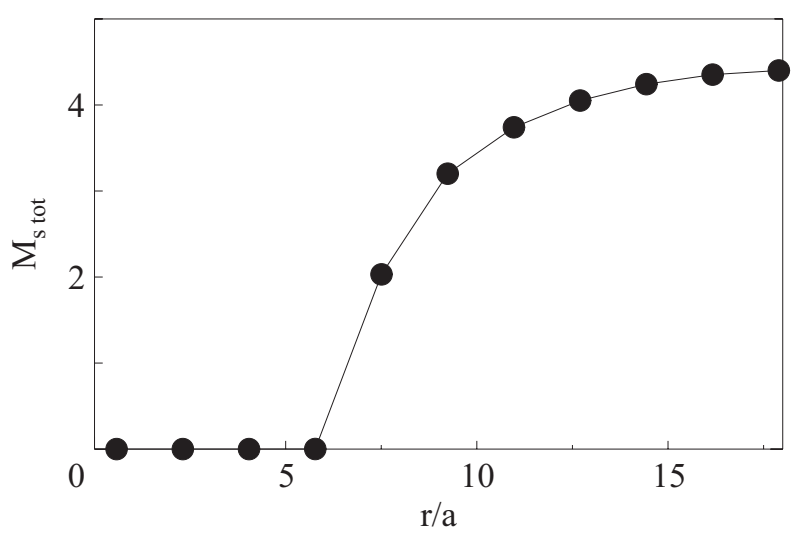

Fig. 5. Dependence of the total sublattice magnetization on the distance $r$ between two vacancies introduced on the different sublattices.

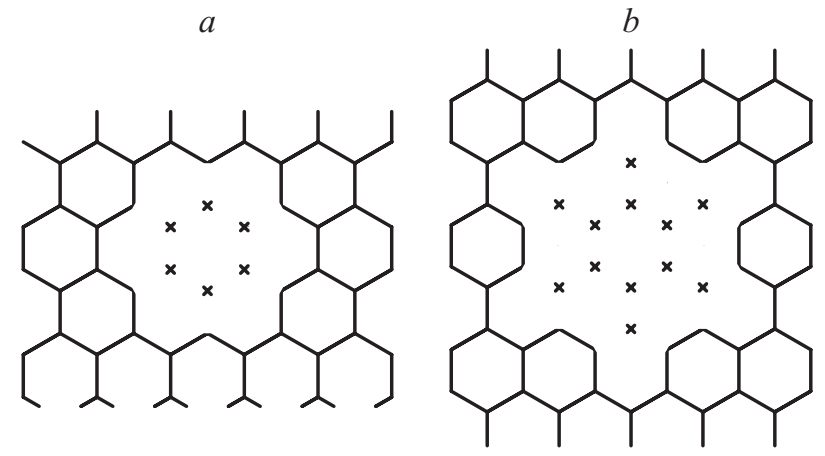

Fig. 6. Examples of extended vacancies inducing no moments around themselves at $U=1.2 t . \quad N_{A}=N_{B}=3 \quad(a)$, and $N_{A}=N_{B}=6(b)$.

The results for two vacancies can be straightforwardly extended to more-than-two vacancies [28]. The point is, as is clear from the above argument, Lieb's theorem. If $N_{A} \neq N_{B}$, local magnetic moments are always induced. When $N_{A}=N_{B}$, the ground state must be a singlet one. If vacancies are close to each other, no local moments is likely to be induced, but if they are wide apart, local moments develop around each vacancy and couple antiferromagnetically with each other. Examples where moments are induced around extended vacancies with $N_{A}=N_{B}$ are shown below.

Cases with $N_{A}=N_{B}$ are shown in Fig. 6. In Fig. 6, $a$, $N_{A}=N_{B}=3$, and in Fig. 6, $b, N_{A}=N_{B}=6$. In both cases, no local moments are formed around the «void» at $U=1.2 t$. Note that these extended vacancies are ring-centered. The spin density in cases with $\left|N_{A}-N_{B}\right| \neq 0$ is shown in Fig. 7. In Fig. 7, $a, N_{A}=7$ and $N_{B}=6$, and in Fig. 7,b, $N_{A}=10$ and $N_{B}=6$. The result for the case with $N_{A}=1$ and $N_{B}=3$ was also previously reported [28]. $a$

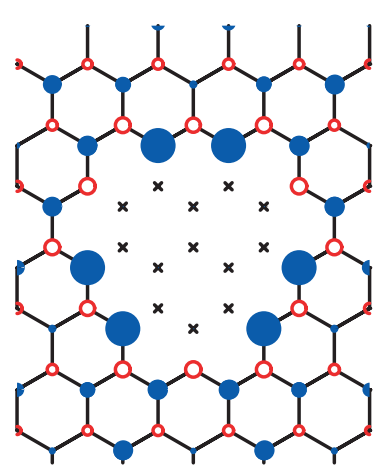

$b$

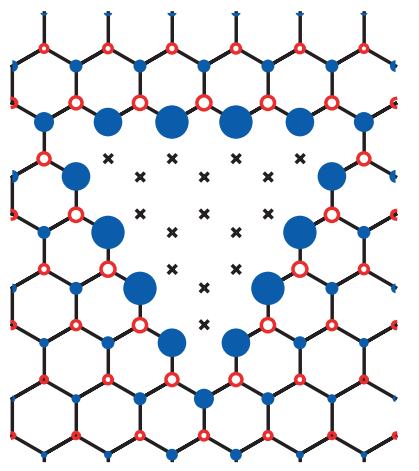

Fig. 7. Spin density around extended vacancies at $U=1.2 t$. $N_{A}=7$ and $N_{B}=6(a)$, and $N_{A}=10$ and $N_{B}=6(b)$. Radius $r_{i}$ of each is dot given by $r_{i}=\sqrt{\left|m_{i}\right|} a$, where $m_{i}$ is the spin density at each lattice point, $m_{i}=\left\langle n_{i} \uparrow-n_{i \downarrow}\right\rangle$. The closed dots represent positive spin density $\left(m_{i}>0\right)$, and open ones represent negative density $\left(m_{i}<0\right)$. 


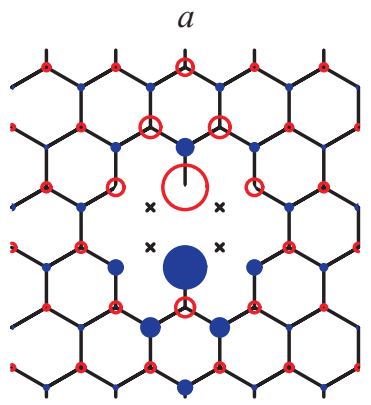

$b$

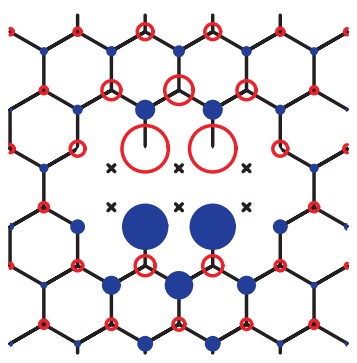

Fig. 8. Spin density around extended vacancies at $U=1.2 t$. $N_{A}=2$ and $N_{B}=2(a)$, and $N_{A}=3$ and $N_{B}=3(b)$. Radius $r_{i}$ of each is dot given by $r_{i}=\sqrt{\left|m_{i}\right|} a$, where $m_{i}$ is the spin density at each lattice point.

These extended vacancies are site-centered. It can be seen that local ferrimagnetic order is induced around the void.

Even when $N_{A}=N_{B}$, it is still possible that local magnetic moments are induced. Examples are shown in Fig. 8, where large local moments are induced at Klein edge sites. Here, it is implicitly assumed that the Klein edge site are doubly hydrogenated when the crosses in Fig. 8 stand for vacancies or that the crosses stand for hydrogen chemisorption sites. In Fig. 8, $a$, antiferromagnetic coupling works between one Klein edge site and the other, but it is so weak because no direct exchange interaction exists between these two sites. That is why local moments survive even though $N_{A}=N_{B}$. The same argument also applies to the case in Fig. 8, $b$.

Other examples where large moments are induced even though $N_{A}=N_{B}$ holds are shown in Fig. 9, where

$a$
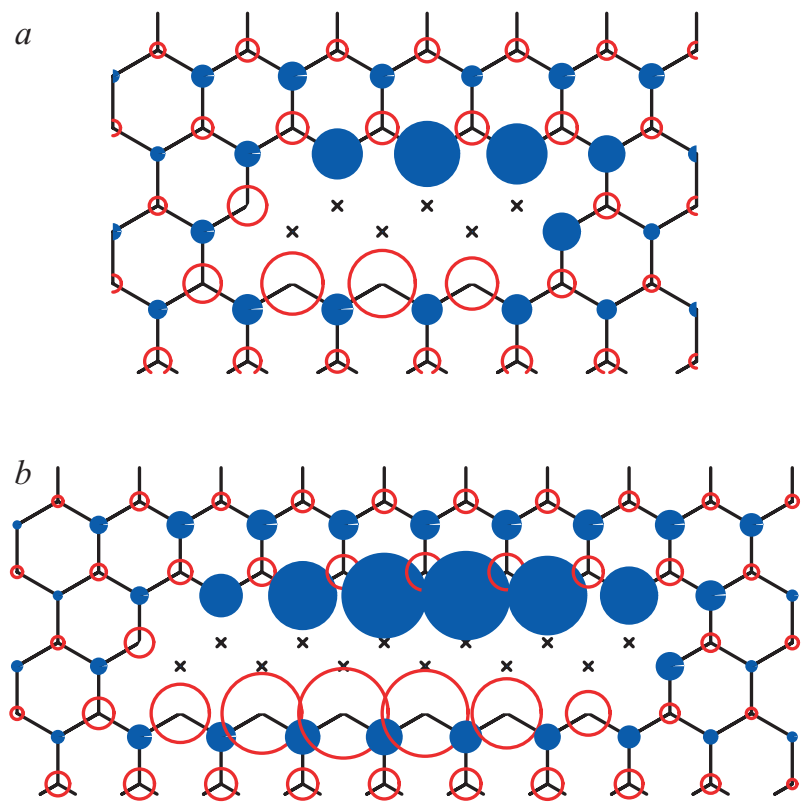

Fig. 9. Spin density around a «slit» with $N_{A}=N_{B}=n_{p}=3$ at $U=1.6 t(a)$, and with $N_{A}=N_{B}=n_{p}=6$ at $U=1.2 t(b)$. Radius $r_{i}$ of each is dot given by $r_{i}=\sqrt{\left|m_{i}\right|} a$, where $m_{i}$ is the spin density at each lattice point. $n_{p}$ pairs of carbon atoms on sublattice $A$ and sublattice $B$ are removed. At $U=1.2 t$, we find no local moments when $n_{p}=1,2,3$, but local moments are found to develop on both sides of the «slit» when $n_{p} \geq 4$. Moments at the edge sites on one side couple ferromagnetically because they are all on the same sublattice, and moments at edge site on different sides couple antiferromagnetically because they belong to the different sublattices. Since there is no direct exchange coupling between the moments on both sides of the slit, local moments remain (The coupling is only through edges of the slit). The true ground state must be a singlet one even in these cases, but the gap between the ground state and the excited state must become small as $n_{p}$ becomes large.

We can also consider a slit consisting vacancies with $N_{A} \neq N_{B}$. An example with $N_{A}=7$ and $N_{B}=6$ is shown in Fig. 10. The ground state must be a triplet one in this case.

We also studied the effect of the next-nearest electron transfer $t^{\prime}$. This transfer directly connects the lattice points on the same sublattice, and the lattice is not bipartite anymore with $t^{\prime}$. At $t^{\prime} \gtrsim 0.2 t$, local moments induced on a zigzag edge start decreasing considerably and diminish at $t^{\prime} \simeq 0.4 t$. The effect of $t^{\prime}$ on other magnetic states is quite similar. This is understood as a result of (1) breaking of particle-hole symmetry resulting in a shift of the energy of quasilocalized states from zero and (2) antiferromagnetic exchange coupling between the next-nearest lattice points caused by $t^{\prime}$. As local moment formation is also obtained with various first-principles calculations, however, magnitude of $t^{\prime}$ is not so large to affect moment formation appreciably.

The charge density is found to be uniform irrespective of the local magnetic moment formation. The nonuniform contribution from the quasilocalized states is cancelled by the contribution from the other occupied extended states [26]. However, this result is a consequence of the neglect of the long-range part of the Coulomb interaction. Adding the repulsive interaction $V$ between the nearest-

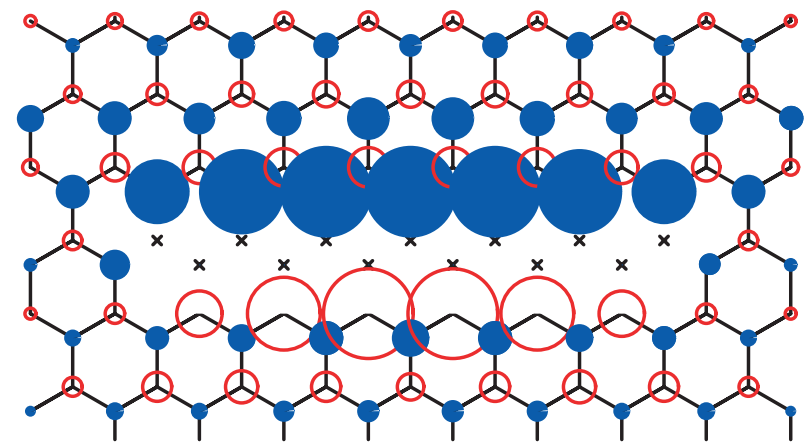

Fig. 10. Spin density around a «slit» at $U=1.2 t . N_{A}=7$ and $N_{B}=6$. Radius $r_{i}$ of each is dot given by $r_{i}=\sqrt{\left|m_{i}\right|} a$, where $m_{i}$ is the spin density at each lattice point. 
neighbor sites to the Hamiltonian and treating it with the mean field theory, we find that the charge density becomes nonuniform around an impurity site. The charge distribution depends on the sign of $u$, the impurity potential. If it is positive, the charge density increases around the impurity site, and it decreases if $u$ is negative. The spin density is not affected substantially by the introduction of the nearesr-neighbor Coulomb interaction as far as $V \lesssim 0.4 U$. It was also argued that the nearest-neighbor Coulomb interaction could cause charge order in graphene [14].

\section{Magnetism induced on edges of graphene}

In Sec. 2 we saw that a zigzag edge is obtained by introducing vacancies on the same sublattice. This implies that localized edge states are associated with a zigzag edge, which favors local moment formation. Indeed, it was found by Fujita et al. that the local ferrimagnetic order is formed near a zigzag edge [8]. Note also that the «slit» introduced in the previous section generates internal zigzag edges in graphene. We can also see that an armchair edge is obtained by removing atoms in a row alternatively from sublattices $A$ and $B$. This implies that no localized states at the chemical potential, and therefore no local magnetic moments, are present near an armchair edge.

A realistic edge is in general a mixture of zigzag and armchair structures [40-43], because it is rather difficult to prepare a graphene sheet with pure zigzag or armchair edges. We then studied the possible magnetism near an edge with structures [31], and found that a zigzag part longer than $3 a$ is sufficient for the formation of local magnetic moments [30,31]. This means, on one hand, that it is rather difficult to suppress magnetism completely on edges of actual graphene sheets. One has to prepare a graphene sheet with a pure armchair edge to make it completely nonmagnetic. On the other hand, it also means that it is fairly easy to make graphene at least locally magnetic.

\section{Summary}

Possible nonmagnetic-defect-induced magnetism in graphene is studied with a tight-binding model. A vacancy (or a hydrogen chemisorption site) is found to induce local magnetic moments around it. In cases of many nonmagnetic defects, the sublattice configuration is essentially important. When the number of vacancies on one sublattice is different from that on the other sublattice, local magnetic moments are necessarily induced. Even when they are equal, it is still possible that local moments are induced.

Our results imply that it is difficult to suppress local magnetism in graphene even when it is completely free from magnetic impurity atoms. On the other hand, the present results also imply that one can control magnetic properties of graphene by controlling defects or impurities. This may be useful in the future application of graphene in spintronic devices. Our results may also be relevant for magnetism of bulk graphite [4-7]. Local moments induced by nonmagnetic defects can lead to bulk ferromagnetism through exchange coupling in 3 dimensions.

The stability of magnetic states or magnetic correlation lenght at finite temperatures [32] is important for its realization. We found that the energy gain due to the local moment formation around a vacancy was approximately $0.01 t(\sim 300 \mathrm{~K})$ at $U=1.0 t$ [27]. As the size of vacancies increases, the energy gain increases more rapidly than linearly. For example, the energy gain of the configuration shown in Fig. 8, $a$ is $0.14 t$ (0.035t per vacancy), and that shown in Fig. 9, $b$ is $0.27 t$ ( $0.023 t$ per vacancy). These results imply that the local magnetic moments persist even at room temperatures.

We used a simple tight-binding model. We always assumed that dangling bonds are terminated by hydrogen atoms, and did not consider the possible lattice distortion induced by vacancies (or chemisorption). Electronic states may depend crucially on the method of termination. Moreover, introduction of defects usually induces lattice distortion. As the size of extended vacancies becomes larger, the effect of lattice distortion will be more important. To study those effects, we have to resort to a firstprinciples calculation. Our results will serve as useful references in those studies.

\section{Acknowledgments}

We would like to thank K. Wakabayashi, S. Okada and O. V. Yazyev for useful discussions and correspondence. This work was partially supported by Research Foundation for the Electrotechnology of Chubu.

1. K.S. Novoselov, A.K. Geim, S.V. Morosov, D. Jiang, Y. Zhang, S.V. Dubonos, I.V. Grigorieva, and A.A. Firsov, Science 306, 666 (2004).

2. A.K. Geim and K.S. Novoselov, Nature Materials 6, 183 (2007).

3. B. Trauzettel, D.V. Bulaev, D. Loss, and G. Burkard, Nature Physics 3, 192 (2007).

4. Carbon-based Magnetism: An Overview of the Magnetism of Metal Free Carbon-bases Compounds and Materials, T.L. Marakova and E. Palacio (eds.), Elsevier Science, Amsterdam (2006).

5. Y. Shibayama, H. Sato, T. Enoki, and M. Endo, Phys. Rev. Lett. 84, 1744 (2000).

6. P. Esquinazi, A. Setzler, R. Höne, C. Semmelhack, Y. Kopelevich, D. Spenmann, T. Butz, B. Kohlstruck, and M. Lösche, Phys. Rev. B66, 024429 (2002). 
7. J. Barzola-Quiquia, P. Esquinazi, M. Rothermel, D. Spemann, T. Butz, and N. Garciá, arXiv: 0711.3542v1 [cond-mat.supr-con].

8. M. Fujita, K. Wakabayashi, K. Nakada, and K. Kusakabe, J. Phys. Soc. Jpn. 65, 1920 (1996).

9. K. Wakabayashi, M. Sigrist, and M. Fujita, J. Phys. Soc. Jpn. 67, 2089 (1998).

10. A.N. Andriotis, M. Menon, R.M. Sheetz, and L. Chernozatonskii, Phys. Rev. Lett. 90, 026801 (2003).

11. K. Kusakabe and M. Maruyama, Phys. Rev. B67, 092406 (2003).

12. S. Okada and A. Oshiyama, J. Phys. Soc. Jpn. 72, 1515 (2003).

13. P.O. Lehtinen, A.S. Foster, A. Ayuela, A. Krasheninnikov, K. Nordlund, and R.M. Nieminen, Phys. Rev. Lett. 91, 017202 (2003).

14. A.Yamashiro, Y. Shimoi, K. Harigaya, and K. Wakabayashi, Phys. Rev. B68, 193410 (2003).

15. Y.H. Kim, J. Choi, K.J. Chang, and S. Tománek, Phys. Rev. B68, 125420 (2003).

16. E.J. Duplock, M. Scheffler, and P.J.D. Linden, Phys. Rev. Lett. 92, 225502 (2004).

17. Y. Ma, P.O. Lehtinen, A.S. Foster, and R.M. Nieminen, New J. Phys. 6, 68 (2004).

18. P.O. Lehtinen, A.S. Foster, Y. Ma, A.V. Krasheninnikov, and R.M. Nieminen, Phys. Rev. Lett. 93, 187202 (2004).

19. Y. Ma, P.O. Lehtinen, A.S. Foster, and R.M. Nieminen, Phys. Rev. B72, 085451 (2005).

20. H. Lee, Y-W. Son, N. Park, S. Han, and J. Yu, Phys. Rev. B72, 174431 (2005).

21. A.N. Andriotis, R.M. Sheetz, E. Richter, and M. Menon, Europhys. Lett. 72, 658 (2005).

22. H. Kumazaki and D.S. Hirashima, J. Phys. Soc. Jpn. 75, 053707 (2006).

23. Y-W. Son, M.L. Cohen, and S.G. Louie, Nature 444, 347 (2006).

24. O.V. Yazyev and L. Helm, Phys. Rev. B75, 125408 (2007).
25. L. Pisani, J.A. Chan, B. Montanari, and N.M. Harrison, Phys. Rev. B75, 064418 (2007).

26. H. Kumazaki and D.S. Hirashima, J. Phys. Soc. Jpn. 76 , 034707 (2007).

27. H. Kumazaki and D.S. Hirashima, J. Phys. Soc. Jpn. 76, 064713 (2007).

28. H. Kumazaki and D.S. Hirashima, Physica E40, 1703 (2008).

29. J. Fernández-Rossier and J.J. Palacios, Phys. Rev. Lett. 99, 177204 (2007).

30. B. Huang, F. Liu, B-L. Gu, and W. Duan, Phys. Rev. B77, 153411 (2008).

31. H. Kumazaki and D.S. Hirashima, J. Phys. Soc. Jpn. 77, 044705 (2008).

32. O.V. Yazyev and M.I. Katsnelson, Phys. Rev. Lett. 100, 047209 (2008).

33. O.V. Yazyev, Phys. Rev. Lett. 101, 037203 (2008).

34. J.J. Palacios, J. Fernández-Rossier, and L. Brey, Phys. Rev. B77, 195428 (2008).

35. K. Wakabayashi, J. Phys. Soc. Jpn. 71, 2500 (2002).

36. V.M. Pereira, F. Guinea, J.M.B. Lopes dos Santos, N.M.R. Peres, and A.H. Castro Neto, Phys. Rev. Lett. 96, 036801 (2006).

37. T.O. Wehling, A.V. Balatsky, K.I. Katsnelson, A.I. Lichtenstein, K. Scharnberg, and R. Wiesendanger, Phys. Rev. Lett. 75, 125425 (2007).

38. E.H. Lieb, Phys. Rev. Lett. 62, 1201 (1989).

39. M. Kohmoto and Y. Hasegawa, Phys. Rev. B76, 205402 (2007).

40. K. Nakada, M. Fujita, G. Dresselhaus, and M.S. Dresselhaus, Phys. Rev. B54, 17954 (1996).

41. Y. Kobayashi, K. Fukui, T. Enoki, K. Kusakabe, and Y. Kaburagi, Phys. Rev. B71, 193406 (2005).

42. Y. Niimi, T. Matsui, H. Kambara, K. Tagami, M. Tsukada, and H. Fukuyama, Phys. Rev. B73, 085421 (2006).

43. Y. Kobayashi, K. Fukui, T. Enoki, and K. Kusakabe, Phys. Rev. B73, 125415 (2006). 